\title{
Understanding the Role of Dipeptidyl Peptidase-4 Inhibitors in COVID-19: Findings From a Systematic Review
}

\author{
Sarah Abdullah Aldukhi ${ }^{\mathrm{a}, \mathrm{c}} \mathbb{0}$, Mohammed Batais ${ }^{\mathrm{b}}$
}

\begin{abstract}
Background: Inhibitors of dipeptidyl peptidase-4 (DPP4) have been used for diabetic patients and there is debate around using the same DPP4 inhibitors as a potential source of treatment for severe coronavirus disease 2019 (COVID-19). The findings from individual studies regarding the usage of DDP4 inhibitors on COVID infection are available but not reviewed and synthesized. Therefore, we aimed to conduct a systematic review to synthesize the findings regarding its effect on the COVID infection course and the outcome.
\end{abstract}

Methods: We carried out an electronic search in Google Scholar and PubMed. We considered a study eligible if it was an original research study, published in English during the era of COVID-19 till September 2021, and had provided information on the topic of DPP4 inhibitors and COVID infection followed by reviewing the full-text articles and summarizing their findings.

Results: This review provided novel insights into the effect of DDP4 inhibitor users and its effect on COVID-19 patients. Overall, literature is scarce on the effect of DDP4 inhibitors on COVID severity and we found mixed results regarding the DDP4 effects on disease severity and mortality. The evidence is not consistent currently as some of the studies supported the role of DDP4 inhibitor, while other studies provided contradictory findings. UK population data published in 2021 with 2.5 million diabetic patients found no evidence to support a protective effect of DDP inhibitors on morbidity and mortality related to COVID-19.

Conclusion: The findings of the review reveal that there is some benefit of the usage of DDP4 inhibitors on COVID-19 morbidity and mortality. However, we highly recommend replicating the studies in different contexts before making any strong recommendations.

Manuscript submitted August 20, 2021, accepted October 12, 2021

Published online November 5, 2021

${ }^{a}$ Department of Family Medicine, King Saud University Medical City, Riyadh, Saudi Arabia

bFamily Medicine, Diabetes \& Chronic Disease Management, Department of Family Medicine, King Saud University Medical City and College of Medicine, KSU, Riyadh, Saudi Arabia

${ }^{\mathrm{c} C}$ Corresponding Author: Sarah Abdullah Aldukhi, Department of Family Medicine, King Saud University Medical City, Riyadh, Saudi Arabia.

Email: SarahAbdullah5@hotmail.com

doi: https://doi.org/10.14740/jem767
Furthermore, the results need to be interpreted cautiously due to the scarcity of literature on the role of DDP4 inhibitors among diabetic patients with COVID-19.

Keywords: DDP4 inhibitors; COVID-19; Diabetes; Systematic review

\section{Introduction}

Since the end of 2019, the novel and deadly infection due to coronavirus disease 2019 (COVID-19) originated in one of the provinces of China followed by its spread throughout the remaining world $[1,2]$. Ever since the first case of COVID-19 was detected in the Hubei district of China (Wuhan) at the end of November 2019, the virus started spreading to both developing and developed countries and regions [3], due to its highly contagious course of disease $[4,5]$. It was only within 3 months after the origin of the virus, the frequency of COVID-19 cases rose to a higher level even outside China, which resulted in reporting of new cases and related deaths to the World Health Organization (WHO) from multiple countries around the world. It was March 16, 2020, when the WHO announced this disease as a pandemic which had then numerous individuals and claimed several lives across the globe [6]. The affected patients can transmit COVID-19 infection to other human beings through direct and indirect contact [7, 8]. Almost the whole world has experienced substantial morbidity and mortality because of the contagious nature of the virus and its associated complications [4, 5]. Furthermore, this unexpected and deadly pandemic has negatively affected the health status, life expectancy, and quality of life of a plethora of human beings on this planet [9]. The highly transmissible and infectious nature of the COVID-19 and its propensity to spread from one human being to the other via aerosol droplets forced countries to impose control and preventive measures $[10,11]$.

Coronaviruses tropism is mainly established by the capability of the spike(s) entry glycoprotein that binds to a receptor on the cell [12]. The existing findings illustrate that coronavirus may use enzymes such as angiotensin-converting enzyme 2, to cause infection among humans [12]. However, the emerging premise has alluded to another receptor, i.e., dipeptidyl peptidase-4 (DPP4) while attaching to the respiratory tract cells. Thus, it seems that the interface between the spike glycoprotein of coronavirus and the human DPP4 also recognized 
as CD26 is a crucial element for virulence, thereby infection among humans [13]. Several clinical and experimental studies over the past three decades have unambiguously indicated that the DPP4/CD26 conduit is implicated in numerous biological mechanisms and illnesses of the immune system [14]. Further, DPP4/CD26 transmembrane glycoproteins are expressed by several cells of the immune system as well as by epithelial and endothelial cells of vessels such as veins, venules, capillaries, and by various organs such as the small bowel, pancreas, kidney, lungs, heart, spleen, liver, and smooth muscle cells [15]. More specifically, it has been reported that cells of the lung and respiratory tract are comparatively rich in DPP4 protein, which is also reflected by the lungs as the most affected organs in the body of humans by COVID-19. This is because the cells within the lung such as pneumocytes embody main cellular locations for coronavirus entrance, and inflammation [16]. Further, DPP4 protein releases the inflammatory markers and cause damage at the cellular level. These results have motivated health care professionals and researchers to use DPP4 protein inhibitors to control cytokine storms resulting from immunomodulators to increase the prognosis and decrease the deaths from COVID-19 [17]. Such inhibitors have been used for diabetic patients and there is debate around using the same DPP4 inhibitors as a potential source of treatment for severe COVID-19 because these inhibitors could suppress T-cell proliferation and the production of pro-inflammatory cytokines. However, the evidence regarding the usage of DDP4 inhibitors on COVID infection is scarce and needs further exploration before establishing their usage for COVID-19 patients. Therefore, we aimed to conduct a systematic review to review and synthesize the findings regarding the mechanisms through which DDP4 works in COVID patients and the outcome comparing patients who have been treated with DDP4 inhibitors and those who have not been treated.

\section{Materials and Methods}

We conducted the systematic review to appraise, synthesize, and aggregate the available evidence to understand the underlying mechanisms through which DDP4 inhibitors prevent the infection or progression of COVID-19. We used PRISMA guidelines to undertake this systematic review and checklist is given in Table 1 [18].

\section{Inclusion and exclusion criteria}

We carried out an electronic search on the different aspects regarding mechanisms of DDP4, its role in the inflammatory process, its linkages with COVID-19, and whether inhibitors of DDP4 could reduce the severity of COVID-19. To address our study question, the eligibility of a study was contingent for inclusion if it was focused on the role of DDP4 on reducing the morbidity and mortality of COVID-19 among diabetic patients since the emergence of COVID-19. Studies that were published in English from 2010 to 2020 across different regions of the world were included. Additionally, only those studies which were quantitative were incorporated. On the contrary, we excluded secondary data, letters to the editor, case reports, and grey literature from this review. The grey literature is defined as any research material that is published by institutions and organizations not included in the commercial or academic publishing channels. The most common grey literature includes technical reports, government documents, abstracts, unpublished data, working papers, dissertation/thesis, patents, and white papers and evaluations [19].

\section{Information sources and search strategy}

A systematic search of published articles was started and completed in 2021. We started and completed a search of published articles that were published during the era of COVID-19 in various journals till September 2021. We explored references of pertinent reviews along with the database searches. An independent search was carried out by two authors who also scanned the results for potentially appropriate studies followed by retrieving the full-text articles. We pre-piloted the search strategies without any restrictions by year of publication, geographic area, or other socio-demographic characteristics.

We searched databases such as TRIP, Cochrane library, PubMed, and MEDLINE. An independent search was carried out by two authors who also scanned the results for potentially appropriate studies followed by retrieving the full-text articles. We grouped search terms into four major categories of the same PICOS (population, intervention, outcome, and settings) framework. The included articles were all English-language studies published in peer-reviewed journals. We found articles relevant to our objectives using the following Medical Subject Heading (MeSH) keywords and text words terms: "DDP4 and COVID-19", "DDP4 inhibitors and COVID-19", "role of DDP4 inhibitors in reducing COVID-19", "benefits of DDP4 inhibitors in reducing the severity of COVID-19", "DDP4 inhibitors and mortality from COVID-19", and "mechanism of DDP4 inhibitors in reducing morbidity from COVID-19. Likewise, the search term for diabetes mellitus (DM) included either "type-2 diabetes mellitus", "diabetes mellitus", "type-2 DM", and "DM". This was followed by combining these major concepts using combinations (AND, OR) relevant to the research question.

\section{Data abstraction}

We imported all appropriate research studies into the reference manager software (EndNote ${ }^{\mathrm{TM}}$ ) file, where each study was reviewed, and we also screened titles for duplicates in this software. We did not consider the abstracts for further review, which did not explicitly explore the study objective. Finally, we obtained and examined the full-text articles of the remaining germane articles. This was followed by abstracting and summarizing the articles that met the eligibility criteria using a standardized proforma. Besides, the bibliography of the remaining studies was also checked and scrutinized to evade missing any useful studies. This process of searching the articles was carried out independently by the reviewers, and their 
Table 1. PRISMA Check List That Was Followed for This Review

\begin{tabular}{|c|c|c|}
\hline Section and topic & Checklist item & Location where item is reported \\
\hline Title & Identify the report as a systematic review. & Abstract, Introduction and Methods \\
\hline Abstract & See the PRISMA 2020 for Abstracts checklist. & Seen and followed this guideline \\
\hline Objectives & $\begin{array}{l}\text { Provide an explicit statement of the objective(s) } \\
\text { or question(s) the review addresses. }\end{array}$ & Objectives are described. \\
\hline $\begin{array}{l}\text { Information } \\
\text { sources }\end{array}$ & $\begin{array}{l}\text { Specify all databases, registers, websites, organizations, reference } \\
\text { lists and other sources searched or consulted to identify studies. } \\
\text { Specify the date when each source was last searched or consulted. }\end{array}$ & $\begin{array}{l}\text { Databases are specified in } \\
\text { Methods section. }\end{array}$ \\
\hline Search strategy & $\begin{array}{l}\text { Present the full search strategies for all databases, registers } \\
\text { and websites, including any filters and limits used. }\end{array}$ & $\begin{array}{l}\text { A full table of search strategy is made } \\
\text { and details are in Methods section. }\end{array}$ \\
\hline $\begin{array}{l}\text { Data collection } \\
\text { process }\end{array}$ & $\begin{array}{l}\text { Specify the methods used to collect data from reports, including } \\
\text { how many reviewers collected data from each report, whether } \\
\text { they worked independently, any processes for obtaining or } \\
\text { confirming data from study investigators, and if applicable, } \\
\text { details of automation tools used in the process. }\end{array}$ & $\begin{array}{l}\text { All required details are in } \\
\text { Methods section. }\end{array}$ \\
\hline \multirow[t]{2}{*}{ Data items } & $\begin{array}{l}\text { List and define all outcomes for which data were sought. Specify } \\
\text { whether all results that were compatible with each outcome domain in } \\
\text { each study were sought (e.g., for all measures, time points, analyses), } \\
\text { and if not, the methods used to decide which results to collect. }\end{array}$ & $\begin{array}{l}\text { All required details are in } \\
\text { Methods section. }\end{array}$ \\
\hline & $\begin{array}{l}\text { List and define all other variables for which data were sought (e.g., } \\
\text { participant and intervention characteristics, funding sources). Describe } \\
\text { any assumptions made about any missing or unclear information. }\end{array}$ & $\begin{array}{l}\text { These are listed in Methods } \\
\text { as well as Tables. }\end{array}$ \\
\hline \multirow{5}{*}{$\begin{array}{l}\text { Synthesis } \\
\text { methods }\end{array}$} & $\begin{array}{l}\text { Describe any methods required to prepare the data for } \\
\text { presentation or synthesis, such as handling of missing } \\
\text { summary statistics, or data conversions. }\end{array}$ & NA \\
\hline & $\begin{array}{l}\text { Describe any methods used to tabulate or visually display } \\
\text { results of individual studies and syntheses. }\end{array}$ & See the flow chart and Tables. \\
\hline & $\begin{array}{l}\text { Describe any methods used to synthesize results and provide a } \\
\text { rationale for the choice(s). If meta-analysis was performed, describe } \\
\text { the model(s), method(s) to identify the presence and extent of } \\
\text { statistical heterogeneity, and software package(s) used. }\end{array}$ & $\begin{array}{l}\text { Since it was not a meta-analysis, } \\
\text { we performed qualitative review. }\end{array}$ \\
\hline & $\begin{array}{l}\text { Describe any methods used to explore possible causes of heterogeneity } \\
\text { among study results (e.g. subgroup analysis, meta-regression). }\end{array}$ & NA \\
\hline & $\begin{array}{l}\text { Describe any sensitivity analyses conducted to assess } \\
\text { robustness of the synthesized results. }\end{array}$ & NA \\
\hline $\begin{array}{l}\text { Reporting bias } \\
\text { assessment }\end{array}$ & $\begin{array}{l}\text { Describe any methods used to assess risk of bias due to missing } \\
\text { results in a synthesis (arising from reporting biases). }\end{array}$ & NA \\
\hline $\begin{array}{l}\text { Certainty } \\
\text { assessment }\end{array}$ & $\begin{array}{l}\text { Describe any methods used to assess certainty (or } \\
\text { confidence) in the body of evidence for an outcome. }\end{array}$ & NA \\
\hline
\end{tabular}


Table 1. PRISMA Check List That Was Followed for This Review - (continued)

\begin{tabular}{|c|c|c|}
\hline Section and topic & Checklist item & Location where item is reported \\
\hline \multirow[t]{2}{*}{ Study selection } & $\begin{array}{l}\text { Describe the results of the search and selection process, from } \\
\text { the number of records identified in the search to the number of } \\
\text { studies included in the review, ideally using a flow diagram. }\end{array}$ & Flow chart and details are in Methods. \\
\hline & $\begin{array}{l}\text { Cite studies that might appear to meet the inclusion criteria, but } \\
\text { which were excluded, and explain why they were excluded. }\end{array}$ & Flow chart and details are in Methods. \\
\hline $\begin{array}{l}\text { Study } \\
\text { characteristics }\end{array}$ & Cite each included study and present its characteristics. & $\begin{array}{l}\text { Description is given in Tables } \\
\text { and Results section. }\end{array}$ \\
\hline \multirow[t]{2}{*}{ Discussion } & Provide a general interpretation of the results in the context of other evidence. & Done. \\
\hline & Discuss any limitations of the evidence included in the review. & Done. \\
\hline
\end{tabular}

judgments and extracted summaries were matched to identify the differences and resolve these accordingly.

Independent reviewers filled a standardized data extraction sheet for the eligible research articles. The reviewers compared the data extraction tables to ensure including the imperative findings of the eligible studies and pilot tested the data extraction sheet before starting the process of data extraction. Besides, prevailing research articles on the chosen topic were reviewed to describe objects of the data extraction sheets. Any discrepancies between the independent reviewers were resolved by consensus between the two reviewers.

\section{Results}

\section{Findings of the search strategy}

We screened the identified articles initially by titles, then by abstracts, and finally, we carried out a full-text articles assessment. We did not include all the articles that did not meet predefined eligibility criteria. As a result, our initial search identified 1,205 citations in different databases; however, 25 articles were duplicates that were removed. Of the remaining 1,180 unique studies, we reviewed titles and abstracts and found 268 relevant abstracts. Upon reviewing abstracts, 249 articles did not meet the eligibility criteria while reviewing the abstracts and eight did not meet eligibility after reviewing full texts. Hence, we were able to retrieve full texts for 11 articles, which were incorporated in the review as shown in Figure 1.

\section{Characteristics of the eligible studies}

With respect to the setting, of these 11 studies, all were observational studies. However, within the observation studies, the majority of them were prospective or retrospective cohorts and only two were case controls and one was case-cross-over. The sample size of all included research studies varied between 85 and 2,796 and all of the studies were conducted in an inpatient setting such as wards but not the outpatient departments. All the studies had included both genders and all studies were published in 2020 (Supplementary Material 1, www.jofem. org) [20-30].

\section{Summary of the main results based on included studies}

Generally, literature is scarce on the effect of DDP4 inhibitors on COVID severity, and below is the synthesis of findings from various epidemiological studies among people with DM. The most important study that initiated the idea about the effect of the role of DDP4 inhibitors in reducing morbidity and mortality due to COVID-19 was conducted in South Korea. This study reported a substantial advantage of DDP4 inhibitor users among diabetic patients with COVID-19. In this study, 263 patients were users of DDP4 inhibitors and 569 were nonusers of DDP4 inhibitors. This study primarily found a lower risk of severe COVID-19 among DDP4 inhibitor users than non-users after adjusting for diverse confounders such as age, gender, hypertension, cardiovascular disease, asthma, and cancer [20], thus highlighting the benefit of DDP4 inhibitor users among diabetic and patients with COVID-19 [20]. The proportion of those who were admitted to ICU due to higher morbidity or had mortality was $3.42 \%$ among users of DDP4 inhibitors and it was $4.39 \%$ among non-users of DDP4 inhibitors. Thus the main findings of the study suggest that DDP4 inhibitors are significantly associated with an improved clinical consequence among patients with COVID-19 as shown in Supplementary Material 1 (www.jofem.org) [20-30].

Another longitudinal study found different risk factors for increased mortality of patients with type $2 \mathrm{DM}$ and COVID-19 [21]. These factors included older age and with 1 year increase in age, the odds of mortality increased by $9 \%(\mathrm{P}=$ $0.001)$. Another significant risk factor of mortality was elevated $\mathrm{C}$-reactive protein $(\mathrm{P}=0.043)$ and the use of insulin $(\mathrm{P}=$ 


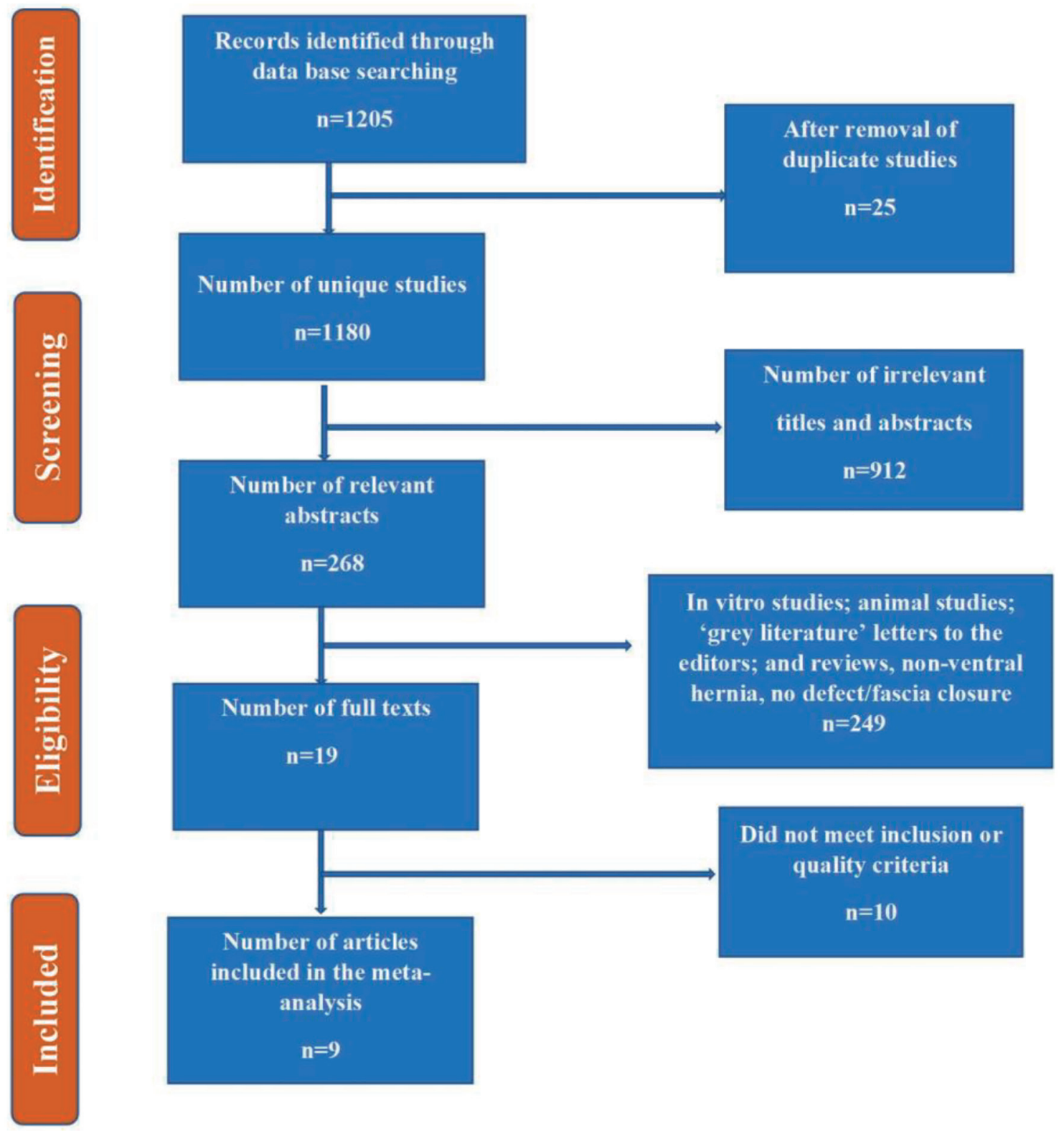

Figure 1. Flow chart summarizing the identification and selection of papers for systematic review.

0.009). Generally, these factors were associated with a worse prognosis and a higher probability of mortality [21]. However, there were no significant differences in the clinical outcomes of those patients who were using medications such as angiotensin-converting enzyme (ACE) inhibitors or angiotensin II receptor blockers (ARBs) vs. those who were not using these medications ( $\mathrm{P} \geq 0.05)$ [21]. However, multivariable regression analyses indicated that none of the glucose-lowering medications (metformin, insulin, a-glycosidase, secretagogues, or DPP4 inhibitors) were associated with in-hospital death (Supplementary Material 1, www.jofem.org) [20-30].

Analogously, another retrospective study was conducted on 889 relatively sick patients, of whom 337 were diagnosed with type 2 DM. This study found that in older males with heart disease, ischemic heart disease with low platelet count and several morbidities were found to be important risk factors for the poor outcomes such as higher mortality (death) and morbidity (indicated by admission to intensive care unit (ICU)) among COVID patients with DM adjusting for confounders such as ethnicity, anemia, temperature, respiratory rate, heart rate, systolic and diastolic blood pressures [30]. More specifically, males were more likely to suffer from COVID-19 associated morbidity and mortality than females with a P-value of 0.031. Likewise, older patients had a higher risk of being admitted to the ICU (morbidity) or were more prone to death than younger patients with a significant P-value of 0.005 [30]. Patients with a lower platelet count had a higher risk of morbidity and mortality associated with COVID-19 with a P- 
value of 0.001 [30]. However, this study did not find any significant differences in mortality after being admitted to ICU for 1 month. This alludes to the fact that DDP4 inhibitor usage is not significantly associated with mortality among patients with COVID-19 among 337 study participants [30].

Another study was conducted on around 1,317 diabetic patients with COVID-19, who included both DDP4 inhibitors'users and non-users. The main study findings revealed that around one-third of the patients $(29.0 \%)$ experienced the primary outcome of interest, which was a combination of morbidity and mortality such as the risk of intubation and/or death within a week of hospital admission [22]. Around $10.6 \%$ of the patients in this cohort experienced mortality; however, $18.0 \%$ were discharged on day 7 . The final model adjusted for age and sex revealed that body mass index (BMI), patients' age, lymphocyte count, obstructive sleep apnea, micro- and macrovascular complications related to DM were found to be independent risk factors of mortality on day 7 with a P-value $<0.05$ for all these variables [22].

Another multi-center longitudinal study was conducted on 2,796 participants. It was found that $20.6 \%$ of the patients experienced mortality on 28 days of the follow-up. The final multivariable analysis revealed that factors such as younger people who were on routine medications such as metformin therapy with a longer duration of symptoms at the time of admission were less likely to die in the hospital and more likely to discharge from the hospital. In addition, patients with microvascular complications, who were on anticoagulation therapy, had dyspnea at the time of admission, with higher levels of white cell count, C-reactive protein, and higher levels of aspartate aminotransferase were less likely to be discharged and within 28 days than their counterparts with a P-value of $<$ 0.001 for these factors [23]. With respect to the usage of regular medications, patients using metformin and DPP4 inhibitors had a higher probability of discharge, whereas those patients who were using insulin, diuretics, $\beta$-blockers, and anticoagulation medications had a lower chance of discharge [23].

One more retrospective study undertaken on 85 patients with DM and COVID-19 did not find any difference in the rate of admissions in ICU (P-value: 0.329) as well as mortality (Pvalue: 0.821 ) between two groups of people, i.e., users of DDP4 inhibitors and no use of DDP4. For example, the P-values for age, sex, hypertension, and current smoking were $0.647,0.392$, and 0.977 , respectively. However, their analysis was univariate, and the researchers did not adjust for known confounders that could be common causes of disease and exposure [24]. These findings were also endorsed by researchers that conducted a study in Italy and Korea, where authors confirmed no difference between mortality and severity of disease [31,32].

Another study found slightly conflicting results and their results revealed a four times higher risk of being admitted in the ICU among the users of DDP4 inhibitors than their counterparts, those who did not use DDP4 inhibitor. They also found a higher association between DDP4 inhibitors and the risk of admission in ICU [25]. Further, the findings revealed a significant association between comorbidity of DM and hypertension and study outcomes such as hypoxia and admission to ICU (P-value $<0.05$ ) indicating the morbidity. Among diabetics, those who were receiving DDP4 inhibitors were at higher risk of being ad- mitted into the hospital than those who were not with statistically significant results $(\mathrm{P}$-value $<0.05)$. This was further confirmed by a multivariable sensitivity analysis that adjusted for BMI, hemoglobin A1c (HbA1c), systolic, and diastolic blood pressures at the baseline. Further, there was no difference found in the results when these findings were limited to the patients taking metformin [25]. This means that when the findings were limited to patients taking metformin, there was no difference in the findings from the primary findings mentioned above. Patients diagnosed with type 2 DM and who were using DPP4 inhibitors had substantially lower levels of brain-derived neurotrophic factor [25]. However, a case series of around 27 patients reported a lower rate of intubation in DPP4 inhibitor users when compared to the non-users [26]. Further, the study findings revealed that patients admitted to ICU were overweight or obese men suffering from arterial hypertension and treated by antidiabetic drugs and medicines for heart disease.

Similarly, the largest retrospective study found that those who used DDP4 inhibitors such as sitagliptin were less likely to die from all causes than those who did not use it and the results were statistically significant [27].

These findings were also confirmed by another study where it was found that all-cause mortality was reduced among DDP4 inhibitor users than non-users [33]. More specifically, treatment with highly selective DDP4 inhibitors at the time of hospitalization was related to decreased mortality (P-value: 0.0001 ), with significant progress in clinical outcomes (Pvalue: 0.0001$)$ that revealed reduced mortality and with more hospital discharges (P-value: 0.0008) compared with patients not receiving DDP4 inhibitors [33].

Some of the studies were conducted recently in 2021 in the same area. For instance, a study conducted by Wander et al in the USA $(n=35,879)$ found that among diabetic individuals, there was a positive association with prior sulfonylurea or insulin use and adverse outcomes such as hospitalization, ICU admissions, and death at 30 days [28]. However, there was no significant association between DDP4 inhibitors and other antidiabetic medications such as glucagon-like peptide-1 (GLP-1) receptor agonists/sodium-glucose co-transporter-2 (SGLT-2) inhibitors and the same adverse outcomes [28]. The potential confounders that authors considered in their analysis included age, sex, race, smoking, urban/rural status, comorbid, and use of statins [28]. Likewise, recently a nationwide observational cohort study published in 2021 provided by far the most complete data and found no evidence to support a protective effect of DDP inhibitors on the outcomes among diabetic patients with COVID-19 [29]. The authors studied 2,851,465 people with type 2 DM and the findings revealed negligible evidence of an association between DDP inhibitors and COVID-related mortality (hazard ratio (HR): $1.07,95 \%$ confidence interval (CI): $1.01-1.13$ ). These differences in the HR of mortality are minute and are probably due to confounding by indication because these are less likely to be used on older or frail people [29].

\section{Discussion}

This review was undertaken to understand the effect of DPP4 inhibitors on the morbidity and mortality associated with COV- 
ID-19 among diabetic patients. Given the emergence of a new virus, the literature regarding this research area is typically scarce and there is a dearth of literature. Overall, the findings are mixed about the effect of DPP4 inhibitors on the outcome of interest; however, most of the studies showed a positive effect of DPP4 inhibitors on the morbidity and mortality related to COVID-19. However, the existing premise from available studies provides a direction to propose some research questions and hypotheses to be tested in future studies. There are different underlying mechanisms through which DPP4 inhibitors might play a role in reducing morbidity and mortality associated with COVID-19 among diabetic patients. The available literature regarding the effect of DDP4 inhibitors on COVID severity is synthesized and summarized in the following paragraphs.

DPP4 is a commonly expressed ectopeptidase serine membrane-anchored ectopeptidase that survives on the surface of different cells and separates dipeptides from the N-terminus, where a remnant of proline is in the penultimate position [34]. DPP4 acts as a binding protein and a ligand of factors at the extracellular level in addition to its catalytic action, and DPP4 can cleave a substantial number of particles $[35,36]$. Despite the lack of pathological data, it seems that COVID-19 infects the respiratory tract and causes irreversible and fatal pneumonia by inflammatory markers by replicating itself. The replication of the virus can result in delayed interferon response, infiltration by neutrophils and macrophages, and also the production of inflammatory cytokines and chemokines [37]. Furthermore, there is evidence of vascular leakage that causes alveolar edema and collapse [37].

In addition, the existing literature has highlighted several mechanisms through which DDP4 inhibitors could reduce morbidity and mortality among COVID-19 patients. For example, one of such mechanisms is by reducing the inflammatory response at the cellular level by reducing the inflammatory markers such as NLRP3 (NOD-like receptor 3) inflammasome, C-reactive protein, tumor necrosis factor- $\alpha$, and interleukin-6 (IL-6), IL-1b, and IL-18 [38-42]. Moreover, the literature also alludes to the role of decrease in mRNA expression of CD26 mainly in the mononuclear cells. There is also evidence of suppressing the myelin basic protein-specific CD4b T-cell clones and blocking the path through which transforming growth factor (TGF)- $\beta$ induces the activation of fibroblasts. Above all, DDP4 inhibitors have been found to reduce the activity of DDP4 among older individuals, obese patients, and patients with type 2 DM. This mechanism in turn will result in the reduction of inflammation among these groups of patients. Lastly, the literature also highlights that a rise in soluble DPP4/CD26 levels tends to keep the COVID-19 virus away from entering the targeted cells [38-42]. Summarizing altogether, this premise indicates that there may be an impact of COVID-19 on tissues that are modulated by DPP4. Therefore, inhibition of DPP4 might block these mechanisms and such an approach could be helpful to devise the appropriate treatment of COVID-19 [43, 44].

\section{Strengths and limitations}

This review is the first of its kind that could be a gateway to understand the effect of DDP4 inhibitors on morbidity and mortality associated with COVID-19. We followed a systematic approach and conducted this review under standard guidelines that are universally used. Given the novice virus and its effect on the world, researchers are still exploring this phenomenon further. For example, negligible studies found the individual effect of different types of DDP4 inhibitors on the outcome. Therefore, there is a need to investigate the effect of different types of DDP4 inhibitors on morbidity and mortality on COVID-19. Secondly, none of the studies explored the side effects of DDP4 inhibitors and their safety among the patients. Therefore, it is recommended to carry out research in that direction to weigh the risks and benefits of DDP4 inhibitors on COVID-19 morbidity and mortality. Since COVID-19 is an emerging area of interest with a novice relationship with DM and DDP4 inhibitors, there is a dearth of literature on different types of DDP4 inhibitors and their effects on COVID-19 outcomes. Therefore, we recommend studying and comparing different types of DDP4 and their effects on COVID outcomes.

\section{Conclusion and Recommendation}

This review provided novel insights into the effect of DDP4 inhibitor users and its effect on COVID-19 patients. Generally, we found mixed evidence regarding the effect of DDP4 inhibitor users on COVID-19 and its severity also mortality from COVID-19. The evidence is not consistent currently as some of the studies provided evidence of DDP4 inhibitor users on COVID-19 mortality and morbidity, while other studies provided opposite evidence. This reveals that this field is emerging, and we need more evidence about the usage of DDP4 inhibitors on COVID-19 morbidity and mortality. Moreover, the studies conducted so far are from developed countries than developing countries and we highly recommend replicating these studies in the context of developing countries before making any strong recommendations to widely use DDP4 inhibitors among diabetic patients with COVID-19. Further, the studies which reported the benefit of use of DDP4 inhibitors among diabetic patients did not control for all potential confounders such as older age, chronic kidney, and cardiovascular disease, and other comorbidities. This means that the effect of DDP4 inhibitors could be confounded due to common causes of DDP4 inhibitors and COVID-19 morbidity and mortality, therefore, we need to interpret the results of positive findings cautiously. Moreover, even though the benefits were found for diabetic patients, the results of the studies did not allude to whether these findings could be generalized to non-diabetic patients or not. Lastly, it is important to understand the underlying mechanisms through which DDP4 inhibitors improve the outcomes among COVID-19 patients even though few mechanisms are cited in the literature.

\section{Supplementary Material}

Suppl 1. Characteristics of the Studies Included in the Systematic Review With the Main Findings and Summary of Key Findings $(n=11)$. 


\section{Acknowledgments}

None to declare.

\section{Financial Disclosure}

This research did not receive any specific grant from funding agencies in the public, commercial, or not-for-profit sectors.

\section{Conflict of Interest}

The authors declare that there is no conflict of interest.

\section{Author Contributions}

SAA conducted the research, data analysis, and write the manuscript. MB supervised her during the research and did the final editing of the manuscript. Both authors agreed on the final draft of the manuscript for submission and publication.

\section{Data Availability}

The authors declare that data supporting the findings of this study are available within the article.

\section{References}

1. Chen Y, Liu Q, Guo D. Emerging coronaviruses: Genome structure, replication, and pathogenesis. J Med Virol. 2020;92(4):418-423.

2. Tu H, Tu S, Gao S, Shao A, Sheng J. The epidemiological and clinical features of COVID-19 and lessons from this global infectious public health event. J Infect. 2020;81(1):1-9.

3. Liu SL, Saif L. Emerging viruses without borders: the Wuhan coronavirus. Viruses. 2020;12(2):130.

4. Nishiura H, Jung SM, Linton NM, Kinoshita R, Yang Y, Hayashi K, Kobayashi T, et al. The extent of transmission of novel coronavirus in Wuhan, China, 2020. J Clin Med. 2020;9(2).

5. Khachfe HH, Chahrour M, Sammouri J, Salhab H, Makki BE, Fares M. An Epidemiological Study on COVID-19: A Rapidly Spreading Disease. Cureus. 2020;12(3):e7313.

6. Mahase E. Covid-19: WHO declares pandemic because of "alarming levels" of spread, severity, and inaction. BMJ. 2020;368:m1036.

7. Khurshid Z, Asiri FYI, Al Wadaani H. Human saliva: non-invasive fluid for detecting novel coronavirus (2019nCoV). Int J Environ Res Public Health. 2020;17(7):2225.

8. Peng X, Xu X, Li Y, Cheng L, Zhou X, Ren B. Transmission routes of 2019-nCoV and controls in dental practice. Int J Oral Sci. 2020;12(1):9.

9. Sjodin H, Wilder-Smith A, Osman S, Farooq Z, Rocklov
J. Only strict quarantine measures can curb the coronavirus disease (COVID-19) outbreak in Italy, 2020. Euro Surveill. 2020;25(13):2000280.

10. Talevi D, Socci V, Carai M, Carnaghi G, Faleri S, Trebbi E, di Bernardo A, et al. Mental health outcomes of the CoViD-19 pandemic. Riv Psichiatr. 2020;55(3):137-144.

11. McKibbin WJ, Fernando R. The global macroeconomic impacts of COVID-19: Seven scenarios. 2020.

12. Zhou P, Yang XL, Wang XG, Hu B, Zhang L, Zhang W, Si $\mathrm{HR}$, et al. A pneumonia outbreak associated with a new coronavirus of probable bat origin. Nature. 2020;579(7798):270273.

13. Vankadari N, Wilce JA. Emerging WuHan (COVID-19) coronavirus: glycan shield and structure prediction of spike glycoprotein and its interaction with human CD26. Emerg Microbes Infect. 2020;9(1):601-604.

14. Waumans Y, Baerts L, Kehoe K, Lambeir AM, De Meester I. The dipeptidyl peptidase family, prolyl oligopeptidase, and prolyl carboxypeptidase in the immune system and inflammatory disease, including atherosclerosis. Front Immunol. 2015;6:387.

15. Meyerholz DK, Lambertz AM, McCray PB, Jr. Dipeptidyl peptidase 4 distribution in the human respiratory tract: implications for the Middle East respiratory syndrome. Am J Pathol. 2016;186(1):78-86.

16. Zaki AM, van Boheemen S, Bestebroer TM, Osterhaus $\mathrm{AD}$, Fouchier RA. Isolation of a novel coronavirus from a man with pneumonia in Saudi Arabia. N Engl J Med. 2012;367(19):1814-1820.

17. Maddaloni E, Buzzetti R. Covid-19 and diabetes mellitus: unveiling the interaction of two pandemics. Diabetes Metab Res Rev. 2020;36(7):e33213321.

18. Moher D, Liberati A, Tetzlaff J, Altman DG, The PRISMA Group. Preferred reporting items for systematic reviews and meta-analyses: the PRISMA statement. Int J Surg. 2010;8(5):336-341.

19. Mahood Q, Van Eerd D, Irvin E. Searching for grey literature for systematic reviews: challenges and benefits. Res Synth Methods. 2014;5(3):221-234.

20. Rhee SY. Effects of a DPP-4 inhibitor and RAS blockade on clinical outcomes of patients with diabetes and COVID-19 (Diabetes Metab J 2021;45:251-9). Diabetes Metab J. 2021;45(4):619-620.

21. Chen Y, Yang D, Cheng B, Chen J, Peng A, Yang C, Liu C, et al. Clinical characteristics and outcomes of patients with diabetes and COVID-19 in association with glucose-lowering medication. Diabetes Care. 2020;43(7):1399-1407.

22. Cariou B, Hadjadj S, Wargny M, Pichelin M, Al-Salameh A, Allix I, Amadou C, et al. Phenotypic characteristics and prognosis of inpatients with COVID-19 and diabetes: the CORONADO study. Diabetologia. 2020;63(8):15001515 .

23. Wargny M, Potier L, Gourdy P, Pichelin M, Amadou C, Benhamou PY, Bonnet JB, et al. Predictors of hospital discharge and mortality in patients with diabetes and COVID-19: updated results from the nationwide CORONADO study. Diabetologia. 2021;64(4):778-794.

24. Fadini GP, Morieri ML, Longato E, Bonora BM, Pinelli S, Selmin E, Voltan G, et al. Exposure to dipeptidyl-pepti- 
dase-4 inhibitors and COVID-19 among people with type 2 diabetes: A case-control study. Diabetes Obes Metab. 2020;22(10):1946-1950.

25. Dalan R, Ang LW, Tan WYT, Fong SW, Tay WC, Chan YH, Renia L, et al. The association of hypertension and diabetes pharmacotherapy with COVID-19 severity and immune signatures: an observational study. Eur Heart J Cardiovasc Pharmacother. 2021;7(3):e48-e51.

26. Montastruc F, Romano C, Montastruc JL, Silva S, Seguin T, Minville V, Georges B, et al. Pharmacological characteristics of patients infected with SARS-Cov-2 admitted to Intensive Care Unit in South of France. Therapie. 2020;75(4):381-384.

27. Solerte SB, D'Addio F, Trevisan R, Lovati E, Rossi A, Pastore I, Dell'Acqua M, et al. Sitagliptin treatment at the time of hospitalization was associated with reduced mortality in patients with type 2 diabetes and COVID-19: a multicenter, case-control, retrospective, observational study. Diabetes Care. 2020;43(12):2999-3006.

28. Wander PL, Lowy E, Beste LA, Tulloch-Palomino L, Korpak A, Peterson AC, Young BA, et al. Risk factors for adverse outcomes among 35879 veterans with and without diabetes after diagnosis with COVID-19. BMJ Open Diabetes Res Care. 2021;9(1):e002252.

29. Khunti K, Knighton P, Zaccardi F, Bakhai C, Barron E, Holman N, Kar P, et al. Prescription of glucose-lowering therapies and risk of COVID-19 mortality in people with type 2 diabetes: a nationwide observational study in England. Lancet Diabetes Endocrinol. 2021;9(5):293-303.

30. Izzi-Engbeaya C, Distaso W, Amin A, Yang W, Idowu O, Kenkre JS, Shah RJ, et al. Adverse outcomes in COVID-19 and diabetes: a retrospective cohort study from three London teaching hospitals. BMJ Open Diabetes Res Care. 2021;9(1):e001858.

31. Strollo R, Maddaloni E, Dauriz M, Pedone C, Buzzetti R, Pozzilli P. Use of DPP4 inhibitors in Italy does not correlate with diabetes prevalence among COVID-19 deaths. Diabetes Res Clin Pract. 2021;171:108444.

32. Kim MK, Jeon JH, Kim SW, Moon JS, Cho NH, Han E, You $\mathrm{JH}$, et al. The clinical characteristics and outcomes of patients with moderate-to-severe coronavirus disease 2019 infection and diabetes in Daegu, South Korea. Diabetes Metab J. 2020;44(4):602-613.

33. Mirani M, Favacchio G, Carrone F, Betella N, Biamonte E, Morenghi E, Mazziotti G, et al. Impact of comorbidities and glycemia at admission and dipeptidyl peptidase 4 inhibitors in patients with type 2 diabetes with COV-
ID-19: a case series from an academic hospital in Lombardy, Italy. Diabetes Care. 2020;43(12):3042-3049.

34. Lambeir AM, Durinx C, Scharpe S, De Meester I. Dipeptidyl-peptidase IV from bench to bedside: an update on structural properties, functions, and clinical aspects of the enzyme DPP IV. Crit Rev Clin Lab Sci. 2003;40(3):209294.

35. Lei Y, Hu L, Yang G, Piao L, Jin M, Cheng X. Dipeptidyl peptidase-IV inhibition for the treatment of cardiovascular disease- recent insights focusing on angiogenesis and neovascularization. Circ J. 2017;81(6):770-776.

36. Nistala R, Savin V. Diabetes, hypertension, and chronic kidney disease progression: role of DPP4. Am J Physiol Renal Physiol. 2017;312(4):F661-F670.

37. Channappanavar R, Fehr AR, Vijay R, Mack M, Zhao J, Meyerholz DK, Perlman S. Dysregulated type i interferon and inflammatory monocyte-macrophage responses cause lethal pneumonia in SARS-CoV-infected mice. Cell Host Microbe. 2016;19(2):181-193.

38. Nauck MA, Meier JJ. Reduced COVID-19 mortality with sitagliptin treatment? Weighing the dissemination of potentially lifesaving findings against the assurance of high scientific standards. Diabetes Care. 2020;43(12):29062909.

39. Kagal UA, Angadi NB, Matule SM. Effect of dipeptidyl peptidase 4 inhibitors on acute and subacute models of inflammation in male Wistar rats: An experimental study. Int J Appl Basic Med Res. 2017;7(1):26-31.

40. Birnbaum Y, Bajaj M, Qian J, Ye Y. Dipeptidyl peptidase-4 inhibition by Saxagliptin prevents inflammation and renal injury by targeting the Nlrp3/ASC inflammasome. BMJ Open Diabetes Res Care. 2016;4(1):e000227.

41. Kawasaki T, Chen W, Htwe YM, Tatsumi K, Dudek SM. DPP4 inhibition by sitagliptin attenuates LPS-induced lung injury in mice. Am J Physiol Lung Cell Mol Physiol. 2018;315(5):L834-L845.

42. Liu X, Zhang T, Zhang C. Sitagliptin inhibits extracellular matrix accumulation and proliferation in lung fibroblasts. Med Sci Monit. 2020;26:e922644.

43. Klemann C, Wagner L, Stephan M, von Horsten S. Cut to the chase: a review of CD26/dipeptidyl peptidase-4's (DPP4) entanglement in the immune system. Clin Exp Immunol. 2016;185(1):1-21.

44. Trzaskalski NA, Fadzeyeva E, Mulvihill EE. Dipeptidyl peptidase-4 at the interface between inflammation and metabolism. Clin Med Insights Endocrinol Diabetes. 2020;13:1179551420912972. 